Dom. Cien., ISSN: 2477-8818

Vol. 4, núm.4., oct, 2018, pp. 206-240

El control interno y su influencia en la gestión administrativa del sector público

\title{
El control interno y su influencia en la gestión administrativa del sector público
}

\section{Internal control and its influence on the administrative management of the public sector}

\section{Controle interno e sua influência na gestão administrativa do setor público}

\author{
Walter M. Mendoza-Zamora ${ }^{\mathrm{I}}$ \\ walter.mendoza@unesum.edu.ec \\ Tania Y. García-Ponce II \\ tania.garcia@unesum.edu.ec
}

\author{
María I. Delgado-Chávez ${ }^{\text {III }}$ \\ irasema812@yahoo.com.ar \\ Isabel M. Barreiro-Cedeño IV \\ isabel.barreiro@uleam.edu.ec
}

Recibido: 27 de junio de 2018 * Corregido: 28 de agosto de 2018 * Aceptado: 15 de septiembre de 2018

I. Docente de la Universidad Estatal del Sur de Manabí, Gestor Financiero, Ingeniero Comercial, Master en Gestión Empresarial, Docente de la Universidad Técnica Particular de Loja, Loja, Ecuador.

II. Economista, Master en contabilidad y auditoría, Docente de la Universidad Estatal del Sur de Manabí, Jipijapa, Ecuador.

III. Ingeniera en Contabilidad y Auditoría, Master en Tributación y Finanzas, Docente de la Universidad Laica Eloy Alfaro de Manabí, Manta, Ecuador.

IV. Ingeniería en Administración de Empresas, Diplomado por competencias, Docente Universidad Laica Eloy Alfaro de Manabí Manta, Ecuador. 


\section{Resumen}

El control interno ha sido reconocido como una herramienta para que la dirección de todo tipo de organización, obtenga una seguridad razonable para el cumplimiento de sus objetivos institucionales y esté en capacidad de informar sobre su gestión a las personas interesadas en ella. La Gestión de Administración del sector público es la institución integrada por los organismos que conforman la Rama Ejecutiva del Poder Público y por todos los demás organismos y entidades de naturaleza pública que de manera permanente tienen a su cargo el ejercicio de las actividades y funciones administrativas o la prestación de servicios públicos del Estado. Para la elaboración del presente artículo se utilizó el método inductivo-deductivo, así mismo se empleó el método analítico-sintético, todo ellos nos lleva a las conclusiones que mejorando el desempeño de la ejecución de los recursos públicos, los mecanismos de control interno que implementen la Gestión administrativa reforzarán los sistemas administrativos vinculados al ciclo de gasto público, lo que redundará en mejores plazos para la formulación, aprobaciones, ejecución y rendición de cuentas de los recursos públicos asignados o generados, cuando se hable de procesos críticos de la entidad solo vincularán a las unidades que intervienen en todo aquello que tenga que ver directamente con uso de recursos, como son los procesos de compras, logística, almacenaje, entre otros.

Palabras clave: Control, Estado, Herramienta, Gestión, Poder, Recursos. 


\begin{abstract}
Internal control has been recognized as a tool for the management of all types of organizations to obtain reasonable assurance for the fulfillment of their institutional objectives and to be able to report their management to the people interested in it. Management of the public sector is the institution comprising the bodies that make up the Executive Branch of Public Power and all other bodies and entities of public nature that are permanently responsible for the exercise of administrative activities or functions. the provision of public services by the State. For the elaboration of this article the inductive-deductive method was used, likewise the analytical-synthetic method was used, all of them lead us to the conclusions that improving the execution performance of public resources, the internal control mechanisms that implement Administrative Management will strengthen the administrative systems linked to the cycle of public spending, which will result in better terms for the formulation, approvals, execution and accountability of public resources allocated or generated, when talking about critical processes of the entity they will only link to the units that intervene in everything that has to do directly with the use of resources, such as purchasing processes, logistics, storage, among others.
\end{abstract}

Keywords: Control, State, Tool, Management, Power, Resources. 


\section{Introducción.}

El control interno se ha convertido últimamente en uno de los pilares en las organizaciones empresariales, debido a que permite observar con claridad la eficiencia y la eficacia de las operaciones que realizan las empresas; así como la confiabilidad de los registros y el cumplimiento de las leyes, normas y regulaciones aplicables y aplicados en los procesos productivos (Ramón, 2001).

Así mismo, cabe resaltar que la empresa que aplique controles internos en sus operaciones, podrá conocer con seguridad la situación real de las mismas, debido a la importancia de tener una planificación que sea capaz de verificar que los controles se cumplan para darle una mejor visión sobre su gestión, además es importante tener un buen sistema de control interno en las empresas debido a lo práctico que resulta al medir la eficiencia y la productividad al momento de implantarlos; en especial si se centra en las actividades básicas que ellas realizan, pues de ello dependen para mantenerse en el mercado. (Chacón, 2002)

Según las normas de control interno del sector público, se considera al control interno como herramienta que abarca el proceso integral efectuado por el titular, funcionarios y servidores de una entidad, diseñado para enfrentar los riesgos y para asegurar que se alcancen sus objetivos gerenciales. La aplicación del control interno requiere de parte de todos ellos, un compromiso, una planificación sistemática y la verificación del cumplimiento de las tareas asignadas (Gobernanza Democrática, 2011). Las compañías en cualquier contexto geográfico-social, necesitan tener un control interno efectivo, que cumpla la función verificadora, pero esencialmente que sea utilizado como una herramienta facilitadora para el mejoramiento de la gestión, es decir, de la obtención de eficiencia, economía, efectividad y mejora continua.

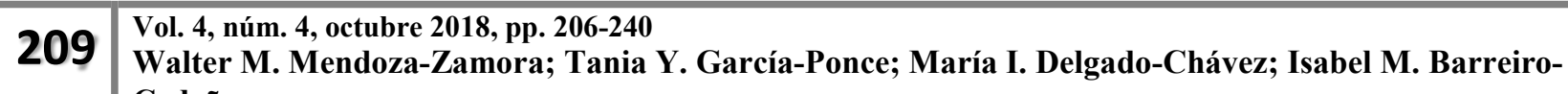
Cedeño
} 
(Catágora, 2013) Las crisis financieras, quiebras emblemáticas de reconocidas empresas comerciales, fraudes ineludibles, estados financieros manipulados; en el plano internacional y nacional han demostrado en la historia que una de sus causas fue la ausencia e insuficiente funcionamiento de los controles internos idóneos sobre las operaciones e información financiera que debieron asegurar el correcto uso de los recursos aportados por los dueños en el sector privado y por los ciudadanos en el caso del sector público.

En este trabajo de investigación se estima que el seguimiento del Control Interno, va a minimizar las deficiencias mejorando los de resultados de la gestión, racionalizar el uso de los recursos propios, que coadyuven el cumplimiento de políticas, planes y normas. De esta forma se mejorará la planeación, organización, dirección y coordinación de la institución; así como la economía, eficiencia y efectividad de los sistemas de administración.

\section{Metodología.}

Para la elaboración del presente artículo se utilizó el método inductivo-deductivo, por cuanto para su elaboración se partió de los elementos conocidos del control interno, su importancia y los principales elementos para poder llegar a lo desconocido, sobre la gestión administrativa del sector público lo que representan las conclusiones.

Así mismo se empleó el método analítico-sintético, ya que se en base a la bibliografía expuesta se analizaron diferentes aspectos documentales para luego sintetizarlos y plasmarlos en el artículo que nos permitimos explicar.

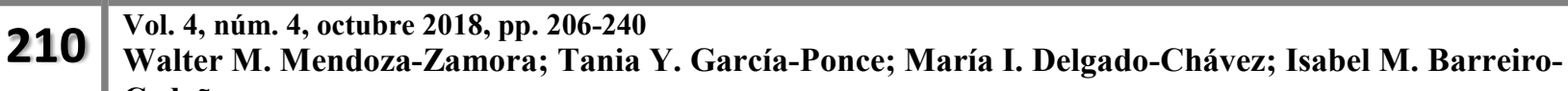
Cedeño
} 


\section{Resultados.}

\section{Definición de Control Interno}

“Control interno" carece de una definición apropiada o universal, o que sea aceptada o aprobada por todos los que investigaron el tema, no existe una completa unificación del significado de la expresión. Sobre el tema se dieron distintas definiciones, provenientes de destacados autores e institucionales nacionales y extranjeras, a continuación se detalla una recopilación de las mismas.

Mejía, (2006), define el control interno como un proceso, ejecutado por la junta de directores, la administración principal y otro personal de la entidad, diseñado para proveer seguridad razonable en relación con el logro de los objetivos de la organización.

Chiavenato, (2004) Sistema de control interno significa, todas las políticas y procedimientos (controles internos) adoptados por la administración de asegurar, hasta donde sea factible la ordenada y eficiente conducción del negocio, incluyendo la adherencia a las políticas de la administración, la salvaguarda de archivos, la prevención y detección de fraudes y errores, la exactitud e integridad de los registros contables y la oportuna preparación de la información financiera confiable.

Chacón, (2002) define como la base sobre el cual descansa la confiabilidad de un sistema contable, el grado de fortaleza determinará si existe una seguridad razonable de las operaciones reflejadas en los estados financieros. Una debilidad importante del control interno, o un sistema de control interno poco confiable, representa un aspecto negativo dentro del sistema contable. Se puede afirmar que un departamento que no aplique controles internos adecuados, puede correr el riesgo de tener desviaciones en sus operaciones, y por supuesto las decisiones tomadas no serán las más

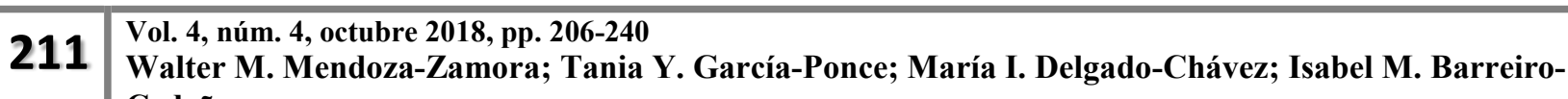
Cedeño
} 
adecuadas para su gestión e incluso podría llevar al mismo a una crisis operativa, por lo que, se debe asumir una serie de consecuencias que perjudican los resultados de sus actividades.

El control interno, entonces, busca promover la eficiencia, eficacia, transparencia y economía en las operaciones de la entidad, la calidad de los servicios públicos que presta, cuidar y resguardar los recursos y bienes del Estado contra cualquier forma de pérdida, deterioro, uso indebido y actos ilegales, en general, contra todo hecho irregular o situación perjudicial que pudiera afectarlos.

\section{Objetivos del Control Interno}

a) Promover y optimizar la eficiencia, eficacia, transparencia y economía en las operaciones de la entidad, así como la calidad de los servicios públicos que presta.

b) Cuidar y resguardar los recursos y bienes del Estado contra cualquier forma de pérdida, deterioro, uso indebido y actos ilegales, así como contra todo hecho irregular o situación perjudicial que pudiera afectarlos.

1. Cumplir la normatividad aplicable a la entidad y sus operaciones.

c) Garantizar la confiabilidad y oportunidad de la información.

d) Fomentar e impulsar la práctica de valores institucionales.

e) Promover el cumplimiento por parte de los funcionarios o servidores públicos de rendir cuenta por los fondos y bienes públicos a su cargo y/o por una misión u objetivo encargado y aceptado. 
f) Promover que se genere valor público a los bienes y servicios destinados a la ciudadanía (Estrada, 2010)

El Control Interno descansa sobre los objetivos antes descritos, si se logra identificar perfectamente cada uno de estos objetivos, se puede afirmar que se conoce el significado de este. En otras palabras toda acción, medida, plan o sistema que emprenda la empresa y que tienda a cumplir cualquiera de estos objetivos, es una fortaleza de Control Interno. Asimismo, toda acción, medida, plan o sistema que no tenga en cuenta estos objetivos o los descuide, es una debilidad de Control Interno, considerado como una herramienta que permite identificar factores de riesgo en ciertas áreas y posibilita lograr un objetivo de control.

Dentro del control interno se pueden determinar cuatro categorías establecidas de objetivos para el control interno, de tipo estratégico, de información financiera, de operaciones y de cumplimiento de las disposiciones legales y reglamentos. Cada una de estas aboga por un tema en específico para lograr ciertas metas de control, no obstante, es el control interno enfocado a la información financiera el que compete y es relevante para las operaciones de auditoría de estados financieros.

\section{Importancia del Control Interno}

El Control Interno trae consigo una serie de beneficios para la entidad, su implementación y fortalecimiento promueve la adopción de medidas que redundan en el logro de sus objetivos. A continuación se presentan los principales beneficios (Estrada, 2010):

La cultura de control favorece el desarrollo de las actividades institucionales y mejora el rendimiento.

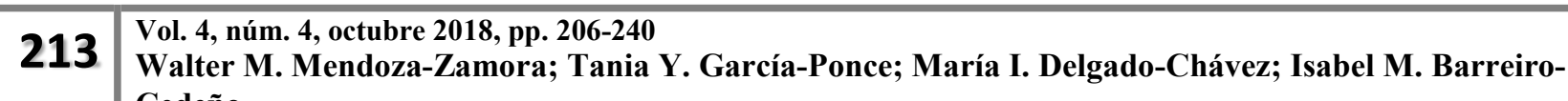
Cedeño
} 
El Control Interno bien aplicado contribuye fuertemente a obtener una gestión óptima, toda vez que genera beneficios a la administración de la entidad, en todos los niveles, así como en todos los procesos, sub procesos y actividades en donde se implemente.

El Control Interno es una herramienta que contribuye a combatir la corrupción.

El Control Interno fortalece a una entidad para conseguir sus metas de desempeño y rentabilidad y prevenir la pérdida de recursos.

El Control Interno facilita el aseguramiento de información financiera confiable y asegura que la entidad cumpla con las leyes y regulaciones, evitando pérdidas de reputación y otras consecuencias.

En conclusión se puede establecer que el control interno ayuda a una entidad a cumplir sus metas, evitando peligros no reconocidos y sorpresas a lo largo del camino.

La implementación y fortalecimiento de un adecuado Control Interno promueve entonces:

a) La adopción de decisiones frente a desviaciones de indicadores.

b) La mejora de la Ética Institucional, al disuadir de comportamientos ilegales e incompatibles.

c) El establecimiento de una cultura de resultados y la implementación de indicadores que la promuevan.

d) La aplicación, eficiente, de los planes estratégicos, directivas y planes operativos de la entidad, así como la documentación de sus procesos y procedimientos. 
e) La adquisición de la cultura de medición de resultados por parte de las unidades y direcciones.

f) La reducción de pérdidas por el mal uso de bienes y activos del Estado.

g) La efectividad de las operaciones y actividades.

h) El cumplimiento de la normativa.

i) La salvaguarda de activos de la entidad (Martillo, 2008).

Tomando en consideración que el control interno es una herramienta surgida de la imperiosa necesidad de accionar proactivamente a los efectos de suprimir y/o disminuir significativamente la multitud de riesgos a las cuales se hayan afectadas los distintos tipos de organizaciones, sean estos privados o públicos, con o sin fines de lucro, siendo la base donde descansan las actividades y operaciones de una entidad; es decir, que las actividades de producción, distribución, financiamiento, administración, entre otras, son regidas por el Control Interno; además, es un instrumento de eficiencia y no un plan que proporciona un reglamento tipo policíaco o de carácter tiránico.

Desarrollar un Control Interno adecuado a cada tipo de organización nos permitirá optimizar la utilización de recursos con calidad para alcanzar una adecuada gestión financiera y administrativa en este caso en el sector público, logrando mejores niveles de productividad.

\section{Funciones del Control Interno}

El Control Interno puede definirse como el plan mediante el cual una organización establece principios, métodos y procedimientos que coordinados y unidos entre sí, buscan proteger los

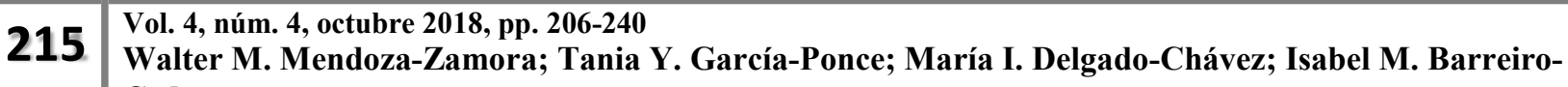
Cedeño
} 
recursos de la entidad y prevenir y detectar fraudes y errores dentro de los diferentes procesos desarrollados en la empresa. Además, hace parte de un proceso que debe ser ejecutado de forma conjunta por la junta directiva de la empresa, la gerencia y por todo el recurso humano de la misma, cuya orientación debe estar encaminada al cumplimiento de las siguientes funciones (Poch, 2000):

1.- Proteger los recursos de la organización, buscando su adecuada administración ante posibles riesgos que los afecten.

2.- Garantizar la eficacia y la eficiencia en todas las operaciones promoviendo y facilitando la correcta ejecución de las funciones y actividades definidas para el logro de la misión de la organización.

3.- Velar porque todas las actividades y recursos de la organización estén dirigidos al cumplimiento de los objetivos de la entidad.

4.- Garantizar la correcta evaluación y seguimiento de la gestión organizacional.

5.- Asegurar que los registros y la información generada como producto de las actividades realizadas por la organización, sean elaborados y presentados de manera oportuno y que dicha información sea confiable.

6.- Definir y aplicar medidas para prevenir los riesgos, así como también detectar y corregir errores que se presenten en la organización y que puedan afectar el logro de sus objetivos.

7.- Garantizar que el Sistema de Control Interno disponga de sus propios mecanismos de verificación y evaluación.

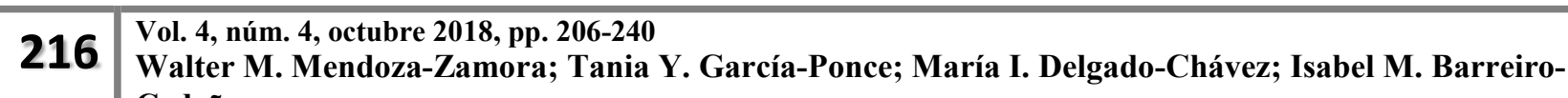
Cedeño
} 
Las funciones del Control Interno tienen por objeto apoyar a la administración en el logro de los objetivos, proporcionando información mediante análisis, apreciaciones y recomendaciones relacionadas con su gestión, es importante tener un buen sistema de control interno en las organizaciones, se ha incrementado en los últimos años, esto debido a lo práctico que resulta al medir la eficiencia y la productividad al momento de implantarlos; en especial si se centra en las actividades básicas que ellas realizan, pues de ello dependen para mantenerse en el mercado; es necesario resaltar, que la empresa que aplique controles internos en sus operaciones, conducirá a conocer la situación real de las mismas, es por eso, la importancia de tener una planificación que sea capaz de verificar que los controles se cumplan para darle una mejor visión sobre su gestión.

\section{Componentes del Control Interno}

Charry-Rodríguez (2013) considera los siguientes componentes del Control Interno:

Entorno (o ambiente) de control.

Proceso de valoración del riesgo de la entidad.

Sistemas de información.

Actividades de control.

Seguimiento (o monitoreo) de los controles. 


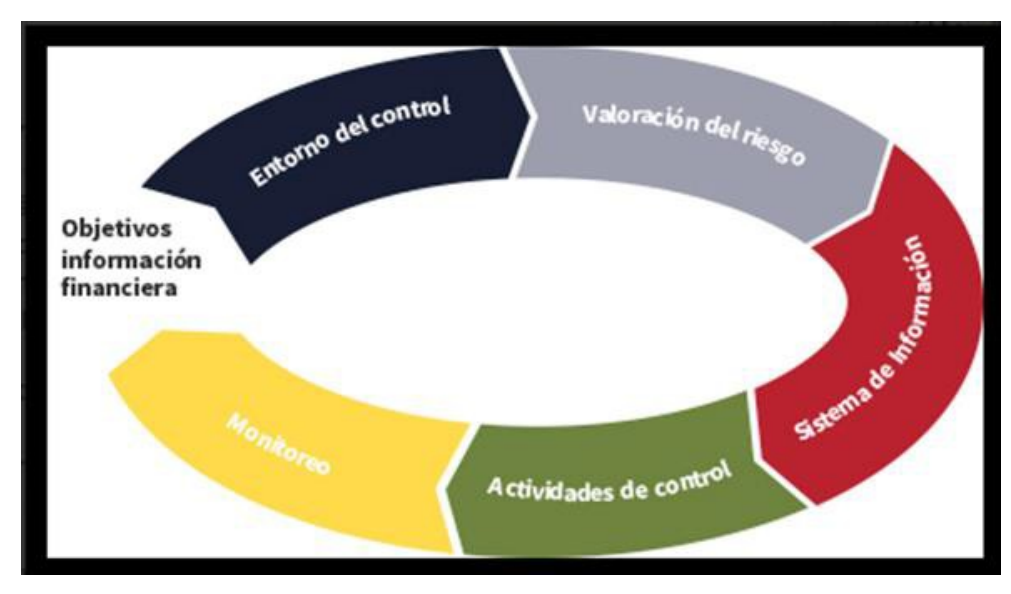

\section{Gráfico 1. Componentes del control interno}

Fuente: (Charry-Rodríguez, 2013)

\section{Entorno (o ambiente) de control}

Este componente es la base para el resto de los componentes del control; un ambiente de control débil origina que sin importar el adecuado diseño del resto de los componentes, no se pueda confiar totalmente en estos. El ambiente de control fija el nivel de disciplina y estructura que hay en la empresa.

Algunas áreas clave al analizar este componente por parte del auditor se enlistan a continuación (Charry, 2013):

Integridad y valores éticos: existe en la empresa desde la alta dirección hasta los niveles iniciales de personal un compromiso con valores de integridad y éticos, tanto en palabras como en hechos, con lo cual se busca desincentivar cualquier tipo de conducta inapropiada.

Compromiso con la competencia: la empresa toma medidas para que su personal operativo y directivo conozca cómo realizar su trabajo de una manera eficiente y adecuada. 
Participación efectiva de los responsables del gobierno de la entidad: existen órganos independientes que efectivamente estén vigilando el adecuado funcionamiento de la empresa.

Estructura organizacional y asignación de autoridad y responsabilidad: existe una estructura organizacional adecuada para llevar a cabo los objetivos, definiéndose los niveles de autoridad y responsabilidad para cada uno de los elementos de esta estructura.

Proceso de valoración de riesgo de la entidad

El componente del proceso de valoración de riesgo de la entidad consiste en que el auditor evalúe lo adecuado del proceso interno de la entidad para identificar los riesgos de negocio de la empresa (relevantes para la información financiera), las estimaciones de la importancia de los mismos, la evaluación de la probabilidad de ocurrencia y la toma de decisiones respecto a dichos riesgos.

El proceso de valoración de riesgo normalmente trata las siguientes cuestiones: cambios en el entorno operativo, nuevas tecnologías, crecimiento rápido, contrataciones de personal de alta dirección, nuevos modelos de negocio, productos o actividades. En caso de que se identifiquen riesgos de incorrección material no identificados por la administración, el auditor deberá cuestionar las razones por las cuales fallaron los procesos de la administración de la empresa para detectarlos y si dichos procesos son adecuados a las circunstancias.

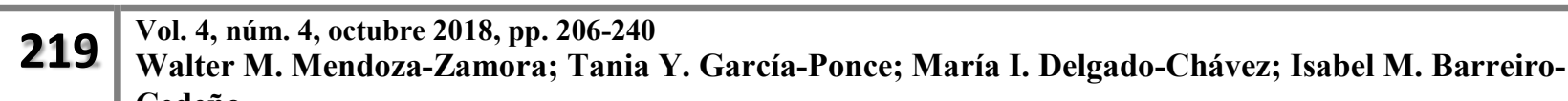
Cedeño
} 
Sistemas de información

Un sistema de información se integra por la infraestructura, software, personas, procedimientos y datos con los que cuenta un negocio o empresa para dirigirla, alcanzar sus objetivos e identificar y responder a los factores de riesgo.

El auditor deberá analizar primordialmente los sistemas de información relacionados con la información financiera; en particular los sistemas relacionados con los procesos operativos (de negocio) tales como: ventas, compras, nóminas, producción, etc.; así como los sistemas de contabilidad que son donde se asientan los registros contables correspondientes.

Al analizar los sistemas de información como parte del proceso de evaluación de los componentes del control interno, deberá considerarse lo siguiente (Fonseca, 2011):

Identificar las fuentes de información utilizadas: en este punto deberán analizarse los tipos de transacciones significativas para los estados financieros, cómo se originan, qué registros contables se generan y cómo captan los sistemas los hechos y condiciones significativos para los estados financieros.

Captación y proceso de información: en este punto deberán identificarse los procesos de información financiera para las transacciones habituales y no habituales, así como la inclusión de estimaciones contables y/o revelaciones significativas.

Utilización de la información generada: En este punto se analizará la forma de comunicar por la empresa la información financiera, los informes resultantes y su utilización en la empresa, así 
como los informes a los responsables del gobierno de la empresa y a terceros, tales como las autoridades regulatorias.

Actividades de control

Las actividades de control son las políticas y procedimientos que ayudan a asegurar que las directrices de la administración se lleven a cabo. Estos controles se refieren a riesgos que, si no se mitigan, pondrían en riesgo el llevar a cabo los objetivos de la empresa.

Las actividades de control pueden clasificarse en los siguientes cuatro tipos (Mantilla, 2008):

Preventivos: controles para evitar errores o irregularidades.

De detección: controles para identificar errores o irregularidades después de que hayan ocurrido para tomar medidas correctivas.

De compensación: controles para brindar cierto grado de seguridad cuando es incosteable la aplicación de otros controles más directos. Ejemplos: segundas firmas, supervisión de terceros, supervisión selectiva interna, etcétera.

De dirección: controles para orientar al personal hacia los objetivos deseados, por ejemplo, las políticas y los procedimientos.

Algunos controles comunes a nivel del proceso operativo incluyen temas como los siguientes (Martillo, 2008):

Segregación de funciones: donde reduce la oportunidad de que una persona por sí misma pueda llevar a cabo u ocultar errores o fraudes.

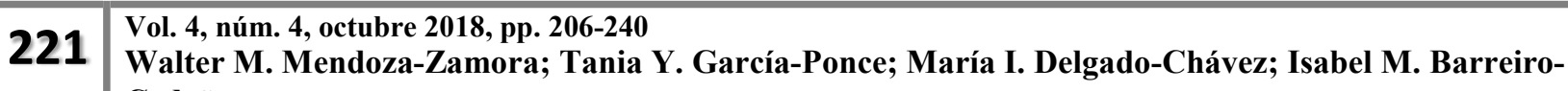
Cedeño
} 
Controles de autorizaciones: define quién tiene la autoridad para aprobar diversas transacciones, comunes o no comunes.

Conciliaciones de cuentas: incluye preparar y revisar conciliaciones oportunamente y tomar decisiones sobre posibles diferencias.

Controles de aplicación de TI: estos se incluyen en las aplicaciones de los sistemas de información, los cuales son automatizados o parcialmente automatizados.

Revisión de resultados reales: comparar los resultados reales contra los presupuestados y periodos anteriores, así como analizar comportamientos inesperados de los resultados.

Controles físicos: están relacionados con la seguridad física de los activos, acceso a instalaciones, registros contables, sistemas de información, archivos de datos, etcétera.

\section{Seguimiento (o monitoreo) de los controles}

El seguimiento o monitoreo evalúa la eficacia de la ejecución del control interno en el tiempo y su objetivo es asegurarse de que los controles trabajen adecuadamente o, en caso contrario, tomar las medidas correctivas necesarias. El seguimiento le permite a la dirección de la empresa saber si los controles internos son eficaces, están implementados adecuadamente, se usan y se cumplen diariamente, o si necesita modificaciones o mejoras (Argandoña, 2012).

El seguimiento se da por la dirección de la empresa, mediante actividades periódicas, evaluaciones específicas o una combinación de ambas. También, el seguimiento de la dirección puede incluir el uso de información externa que pueda resaltar problemas o áreas de oportunidad:

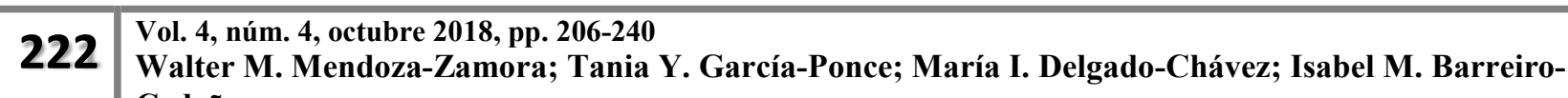
Cedeño
} 
quejas de clientes, comentarios de organismos terceros e informes de auditores externos o consultores sobre al control interno.

Se requiere además de una adecuada planificación para que las tareas, hitos o metas a cumplir se desarrollen en tiempo. El monitoreo y control es un conjunto de actividades de gestión que permiten verificar si el proyecto va marchando según lo planificado. Controla el avance del proyecto en su ejecución, compara el desempeño y mide los resultados reales contra lo planeado, y revisa el comportamiento de los indicadores de desempeño.

El monitoreo y control es una necesidad de cada proyecto para lograr resultados exitosos. Debe hacerse de forma regular y consistente, y debe monitorear la diferencia entre lo planificado y lo real. Es una actividad que muestra cuándo y dónde existieron desviaciones al plan y pone en marcha las acciones correctivas para que el proyecto retorne a su camino normal. Para realizar un control efectivo se deben tener en cuenta dos aspectos fundamentales: la función del control debe centrarse más en prevenir los problemas que en arreglarlos y debe relacionarse tanto con la calidad del producto, como con su cantidad y oportunidad (Charry-Rodríguez, 2013).

Mediante el monitoreo y control se puede comprobar la gestión del alcance, la gestión del tiempo y la gestión del costo, o sea, que el alcance del proyecto se haya establecido correctamente, se examina la programación del proyecto, se revisa la línea base y se controla que se hayan estimado los recursos, en calidad, cantidad y oportunidad, es la acción de verificar que se realicen adecuadamente los reportes previstos para el control del cumplimiento del proyecto, y se valoren los resultados operativos que va teniendo el proyecto durante todo su desarrollo.

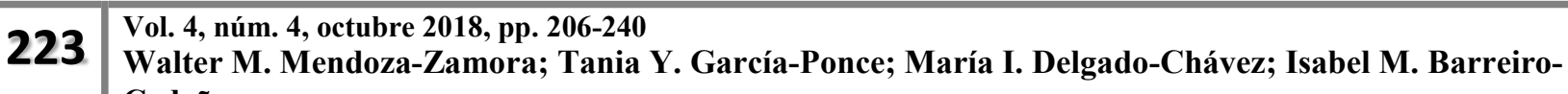
Cedeño
} 
Los componentes del control interno, analizados anteriormente, se categorizan como controles generalizados y de cuentas específicas, los primeros son los que, de manera indirecta, sirven para prevenir que ocurran incorrecciones, o para detectarlas y corregirlas después que hayan ocurrido; los de cuentas específicas están enfocados en riesgos sobre transacciones en particular y diseñados específicamente para prevenir o detectar y corregir incorreciones. Ya que forman parte de la base sobre el cual descansa la confiabilidad de un sistema de gestión administrativa del sector público.

\section{Definición de Gestión Administrativa}

Leonard (2001) explica que administración es la acción de administrar (del latín Administratio - ONIS), acción que se realiza para la consecución de algo o la tramitación de un asunto, es acción y efecto de administrar. Es "la capacidad de la institución para definir, alcanzar y evaluar sus propósitos con el adecuado uso de los recursos disponibles". "Es coordinar todos los recursos disponibles para conseguir determinados objetivos”.

Meigs \& Larsen, (2004) definen el acto de administrar como planeación, organización, dirección y controlar. Las funciones que administración es la acción de administrar (del latín Administratio - ONIS), acción que se realiza para la consecución de algo o la tramitación de un asunto, es acción y efecto de administrar. Es "la capacidad de la institución para definir, alcanzar y evaluar sus propósitos con el adecuado uso de los recursos disponibles". "Es coordinar todos los recursos disponibles para conseguir determinados objetivos".

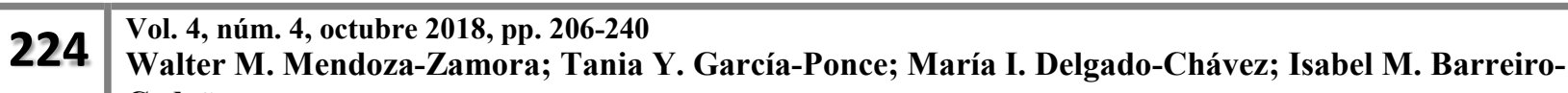
Cedeño
} 
Argandoña (2012) un autor muy trascendente, definió operativamente la administración diciendo que la misma consiste en "Preveer, organizar, mandar, coordinar y controlar, además consideró que era el arte de manejar a los hombres”.

Chiavenato (2004) autor de la Introducción a la Teoría General de la Administración dice: "La palabra administración proviene del latín ad (hacia, dirección, tendencia,) y minister (subordinación u obediencia), y significa aquel que realiza una función bajo el mando de otro, es decir, aquel que presta un servicio a otro." Es "el proceso de planear, organizar, dirigir y controlar el uso de los recursos para lograr los objetivos organizacionales”.

Por lo consiguiente se puede decir que la gestión administrativa en una empresa se encarga de realizar estos procesos recién mencionados utilizando todos los recursos que se presenten en una empresa con el fin de alcanzar aquellas metas que fueron planteadas al comienzo de la misma. En conclusión se trata de un proceso para realizar las tareas básicas de una empresa sistemáticamente, un proceso como la gestión administrativa es mucho más fácil de comprender descomponiéndolo en partes, identificando aquellas relaciones básicas; este tipo de modalidad de descripción se denomina modelo y se utiliza para representar aquellas relaciones complejas.

\section{Importancia de la Gestión Administrativa}

La gestión administrativa en una empresa es uno de los factores más importantes cuando se trata de montar un negocio debido a que de ella dependerá el éxito que tenga dicho negocio o empresa.

Desde finales del siglo XIX se ha tomado la costumbre de definir a la gestión administrativa en términos de cuatro funciones que deben llevar a cabo los respectivos gerentes de una empresa: el

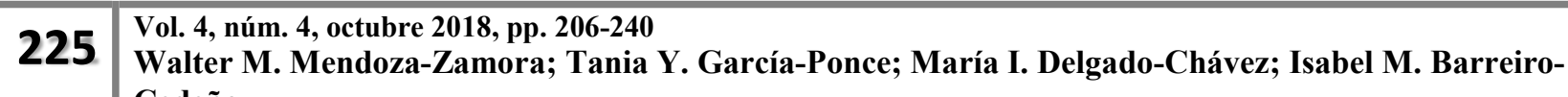
Cedeño
} 
planeamiento, la organización, la dirección y el control, desempeñada para determinar y lograr objetivos manifestados mediante el uso de seres humanos y de otros recursos (Langrod, 2010).

En el sector privado uno de los principales propósitos de la administración financiera es proporcionar información para la toma de decisiones estratégicas. En el Sector Público, sin embargo, la administración financiera todavía se encuentra más orientada al cumplimiento de mandatos legales, basados fundamentalmente en objetivos de políticas públicas, que con la generación de informes adecuados y oportunos para la toma de decisiones (Guerrero, 2008).

En estos argumento, se derivan muchas decisiones claves en la administración financiera del sector público tienden a estar basadas más en la necesidad política que en un análisis cuidadoso de los resultados futuros, esta combinación inevitable de política, leyes y fiscalización pública es lo que hace a la administración financiera gubernamental mucho más difícil y compleja que la administración financiera en el ámbito privado, representando un reto muy particular

En el Sector Público, se requiere de un sistema integrado de administración financiera que permita identificar y disuadir ciertas prácticas irregulares para adoptar medidas correctivas por parte de los organismos fiscalizadores y de control, independientemente de permitir la reprogramación de las actividades planeadas en orden al cumplimiento de los objetivos trazados.

\section{Proceso de la Gestión Administrativa}

Es un conjunto de fases o pasos a seguir para darle solución a un problema administrativo, en él encontraremos asuntos de organización, dirección y control, para resolverlos se debe contar con una buena planeación, un estudio previo y tener los objetivos bien claros para poder hacer del proceso lo más fluido posible (Brever, 2011).

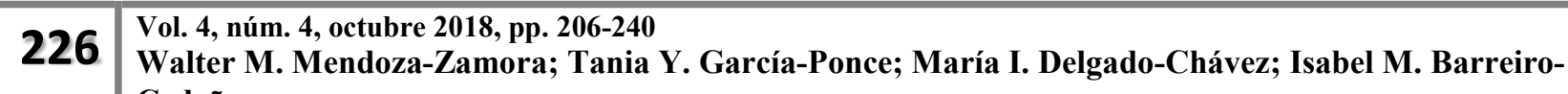
Cedeño
} 
Para que el proceso administrativo llevado a cabo sea el más indicado se deben tomar en cuenta una serie de pasos que no por ser una parte del todo son menos importantes, por mencionar algunos están, las metas, estrategias, políticas, etc.

La técnica de planeación y la organización son partes fundamentales en el proceso ya que ahí radica la complejidad del método que se utilice pues al ser los pasos con más cantidad de deberes se vuelve un poco más complicado tener el control, pero en estos pasos existen más sub-categorías que no deben permitir que el procedimiento se salga de control, esto quiere decir que cada parte tiene una función específica y que si hubiera alguna falla en cualquiera de estos pasos sería difícil lograr un procedimiento limpio y (Rodriguez-Peñaherrera, 2000)

Para una buena gestión pública es prioritario conocer y aplicar el proceso administrativo que implica planificar, organizar, dirigir y controlar adecuadamente la orientación para el trabajo de dirección y liderazgo, es muy importante en el proceso de gestión pública moderna en sí el Modelo de gestión concentra la atención en el resultado de cada uno de los procesos que realiza la Organización, en lugar de las tareas o actividades individuales.

\section{Elementos de la Gestión Administrativa}

Reyes (2012) define el acto de administrar como planeación, organización, dirección y controlar. Las funciones engloban los elementos de la administración. Estos mismos elementos constituyen el proceso administrativo que pueden ser encontrados en cualquier área de la empresa, es decir, que cada cual desempeña actividades de planeación, organización, dirección y control, como actividades administrativas esenciales. A continuación se describe dichos elementos:

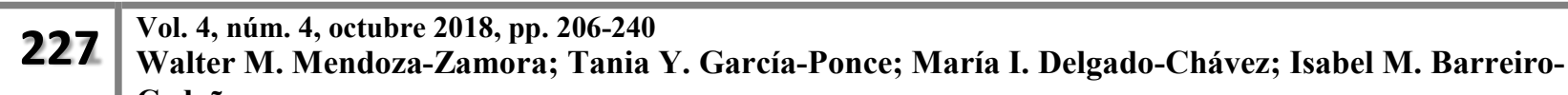
Cedeño
} 
A. Planeación: Consiste en saber qué se va a hacer por anticipado, cuál va a ser la dirección que se va a tomar para alcanzar los objetivos de la manera más eficiente. ¿Qué se desea conseguir (objetivos)? ¿Qué se va a hacer para alcanzarlo? ¿Quién y cuándo lo va a hacer? ¿Cómo lo va a hacer (recursos)? La planeación trata de crear un futuro deseado.

Esta etapa abarca la definición de las metas de la organización, el establecimiento de una estrategia general para alcanzar esas metas y el desarrollo de una jerarquía minuciosa de los planes para integrar y coordinar las actividades; establecer metas sirve para no perder de vista el trabajo que se hará y para que los miembros de la organización fijen su atención en las cosas más importantes (Alvarado, 2012).

Charry-Rodríguez (2013) Afirma que planificar abarca la definición de las metas de la organización, el establecimiento de una estrategia general para alcanzar esas metas y el desarrollo de una jerarquía minuciosa de los planes para integrar y coordinar las actividades. Establecer metas sirve para no perder de vista el trabajo que se hará y para que los miembros de la organización fijen su atención en las cosas más importantes. Elementos de la planeación:

a) Los propósitos: Las aspiraciones fundamentales o finalidades de tipo cualitativo que persigue en forma permanente o semipermanente un grupo social.

b) La investigación: Aplicada a la planeación la investigación consiste en la determinación de todos los factores que influyen en el logro de los propósitos, así como de los medios óptimos para conseguirlos. 
c) Los objetivos: Representan los resultados que la empresa desea obtener, son fines para alcanzar, establecidos cuantitativamente y determinados para realizarse transcurrido un tiempo específico.

d) Las estrategias: Cursos de acción general o alternativas que muestran la dirección y el empleo de los recursos y esfuerzos, para lograr los objetivos en las condiciones más ventajosas.

e) Políticas: Son guías para orientar la acción; son criterios, lineamientos generales a observar en la toma de decisiones, sobre problemas que se repiten dentro de una organización.

f) Programas: Son esquemas en donde se establece, la secuencia de actividades que habrán de realizarse para lograr objetivos y el tiempo requerido para efectuar cada una de sus partes y todos aquellos eventos involucrados en su consecución.

g) Presupuestos: Son los planes de todas o algunas de las fases de actividad del grupo social expresado en términos económicos, junto con la comprobación subsecuente de la realización de dicho plan.

h) Procedimientos: Establecen el orden cronológico y la secuencia de actividades que deben seguirse en la realización de un trabajo repetitivo.

B. Organización: La organización es un sistema que permite una utilización equilibrada de los recursos cuyo fin es establecer una relación entre el trabajo y el personal que lo debe ejecutar. Es un proceso en donde se determina qué es lo que debe hacerse para lograr una finalidad establecida o planeada, dividiendo y coordinando las actividades y suministrando los recursos.

\footnotetext{
\begin{tabular}{l|l}
229 & Vol. 4, núm. 4, octubre 2018, pp. 206-240 \\
Walter M. Mendoza-Zamora; Tania Y. García-Ponce; María I. Delgado-Chávez; Isabel M. Barreiro-
\end{tabular} Cedeño
} 
Chacón, (2002) explica que organizar es disponer el trabajo para conseguir las metas de la organización. Organizar incluye determinar qué tareas hay que hacer, quién las hace, cómo se agrupan, quién rinde cuentas a quién y dónde se toman las decisiones. Elementos de la organización:

- División del trabajo: Para dividir el trabajo es necesario seguir una secuencia que abarca las siguientes etapas: La primera; (jerarquización) que dispone de las funciones del grupo social por orden de rango, grado o importancia. La segunda; (departamentalización) que divide y agrupa todas las funciones y actividades, en unidades específicas, con base en su similitud.

- Coordinación: Es la sincronización de los recursos y los esfuerzos de un grupo social, con el fin de lograr oportunidad, unidad, armonía y rapidez, en el desarrollo de los objetivos.

- Dirección: La dirección es la función que trata, a través de la influencia interpersonal, de lograr que todos los involucrados en la organización contribuyan al logro de sus objetivos. Se ejerce a través de tres subfunciones: el liderazgo, la motivación y la comunicación.

Charry-Rodríguez (2013) dice que la dirección es el proceso para dirigir e influir en las actividades de los miembros de un grupo o una organización entera, con respecto a una tarea. La dirección llega al fondo de las relaciones de los gerentes con cada una de las personas que trabajan con ellos.

La dirección como parte del proceso administrativo se compone de varios elementos:

a) Toma de decisiones. Significa la elección de un curso de acción o alternativa. Al tomar decisiones es necesario antes de evaluar las alternativas, definir y analizar el problema, para posteriormente aplicar la decisión o alternativa que mejor se sugiera.

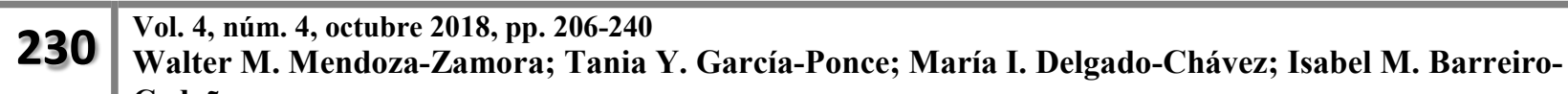
Cedeño
} 
b) Integración. Al igual que en la toma de decisiones, también existe un proceso para la adecuada integración en cuanto al personal se refiere, este proceso inicia con el reclutamiento u obtención de los candidatos que aspiran a un puesto determinado, en seguida se introducirán, o dicho en otras palabras, se les ambientará; para finalmente capacitarlos en el desarrollo de las funciones que habrán de realizar.

c) Motivación. La motivación es la labor más importante de la dirección, a la vez que la más compleja, pues a través de ella se logra la ejecución del trabajo, de acuerdo a normas o patrones de conducta esperados.

d) Comunicación. La comunicación en cualquier grupo que se trate, es de vital importancia ya que involucra a los individuos no solo en su papel de comunicadores, sino en el buen uso que a la información se le da.

e) Supervisión. Consiste en vigilar y guiar a los subordinados de tal forma que las actividades se realicen adecuadamente.

D. Control: El control es la función que efectúa la medición de los resultados obtenidos comparándolos con los esperados (planeados) con el fin de buscar la mejora continua.

Carrasco (2011) sostiene que el control es la actividad de seguimiento encaminada a corregir las desviaciones que puedan darse respecto a los objetivos. El control se ejerce con referencia a los planes, mediante la comparación regular y sistemática de las previsiones y las consiguientes realizaciones y la valoración de las desviaciones habidas respecto de los objetivos. El control, pues, contrasta lo planeado y lo conseguido para desencadenar las acciones correctores, que mantengan el sistema regulado, es decir, orientado a sus objetivos. Elementos del control:

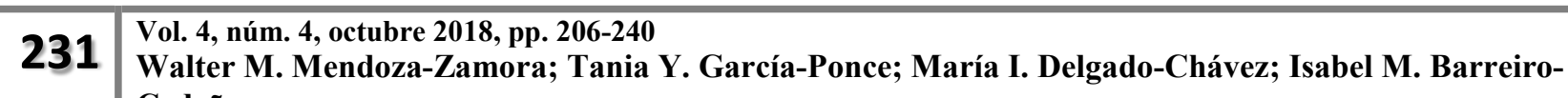
Cedeño
} 
a) Establecimiento de estándares: Es la aplicación de una unidad de medida, que servirá como modelo, guía o patrón con base en lo cual se efectuará el control.

b) Medición de resultados: La acción de medir la ejecución y los resultados, puede de algún modo modificar la misma unidad de medida.

c) Corrección: La utilidad concreta y tangible del control está en la acción correctiva para integrar las desviaciones en relación con los estándares.

d) Retroalimentación: El establecimiento de medidas correctivas da lugar a la retroalimentación; es aquí en donde se encuentra la relación más estrecha entre la planeación y el control.

En fin los elementos de la gestion administrativa sirven para garantizar la disposición de determinadas capacidades y habilidades con respecto a este tema, por ejemplo, la capacidad de liderazgo, la de dirección y en especial, la capacidad de motivación que le puede proporcionar a su equipo de trabajo. Como complemento imprescindible no se puede ignorar el hecho de que en la actualidad para lograr el desarrollo de ciertos elementos mencionados, el responsable de la gestión administrativa de cualquier entidad debe disponer de fundamentos coherentes cuando lleve a cabo la utilización de los recursos económicos, materiales y humanos para el cumplimiento de los objetivos de la misma.

A continuación mediante un gráfico se establecen los elementos de la Gestión administrativa antes descritos:

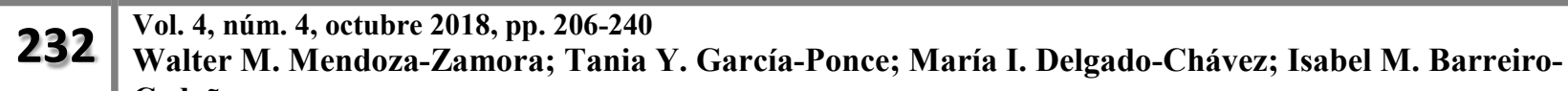
Cedeño
} 


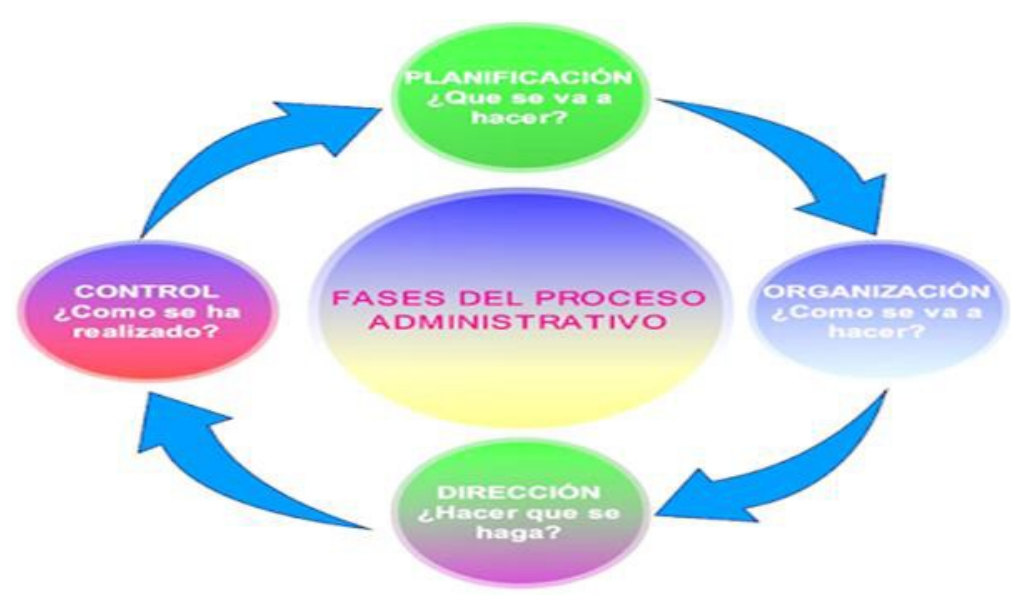

Gráfico 3. Elementos de la Gestión administrativa

Fuente: (Herrera, 2015)

La gestión administrativa trabaja en conjunto con otros departamentos, asegurando de esta forma un desarrollo exitoso en las actividades pertinentes, además de impulsar una organización más eficiente, asimismo, este proceso busca que el empresario sea un verdadero líder de su organización, asegurando la participación de todos que involucrándose en todos los procesos de la cadena productiva. Para ello él debe adquirir compromisos profundos, ya que él es el principal responsable de la ejecución del proceso y la más importante fuerza impulsadora de su empresa.

Para llevar a cabo este proceso tanto en un departamento determinado como en toda organización pública o también privada, se debe tomar en consideración que dicho proceso debe ser: económico, es decir, debe requerir menos esfuerzo que el beneficio que aporta; y acumulativo, que la mejora que se haga permita abrir las posibilidades de sucesivas mejoras a la vez que se garantice el cabal aprovechamiento del nuevo nivel de desempeño logrado. 
Importancia del control interno en la gestión administrativa del sector público

El control interno, lejos de ser un fin en sí mismo que interfiera con el accionar de las entidades, es un medio más para el logro de sus objetivos, por lo tanto, debe entenderse como un proceso que bajo la responsabilidad indelegable de la alta dirección, es ejecutado por ella misma y por todos y cada uno de sus integrantes, esta responsabilidad es de todos los servidores públicos, por lo que se hace necesario que todos conozcan su marco conceptual, sus componentes, la importancia de su implementación y operatividad (Pungitore, 2010).

Se entiende por control interno el sistema integrado por el esquema de organización y el conjunto de los planes, métodos, principios, normas, procedimientos y mecanismos de verificación y evaluación adoptados por una entidad, con el fin de procurar que todas las actividades, operaciones y actuaciones, así como la administración de la información y los recursos, se realicen de acuerdo con las normas constitucionales y legales vigentes dentro de las políticas trazadas por la dirección y en atención a las metas u objetivos previstos (Alvarado, 2012).

En su ejercicio debe contemplar los principios de igualdad, moralidad, eficiencia, economía, celeridad, imparcialidad, publicidad y valoración de costos ambientales, en consecuencia, deberá concebirse y organizarse de tal manera que su ejercicio sea intrínseco al desarrollo de las funciones de todos los cargos existentes en la entidad, y en particular de las asignadas a aquellos que tengan responsabilidad del mando, por lo que se expresará a través de las políticas aprobadas por los niveles de dirección y administración de las respectivas entidades y se cumplirá en toda la escala de la estructura administrativa, mediante la elaboración y aplicación de técnicas de dirección, verificación y evaluación de regulaciones administrativas, de manuales de funciones y procedimientos, de

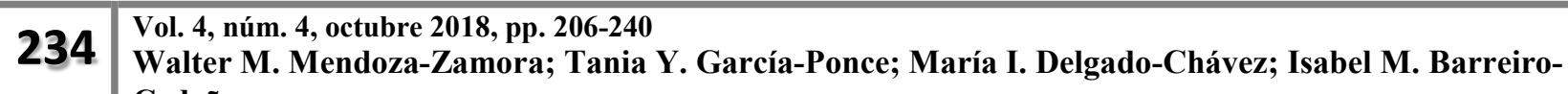
Cedeño
} 
sistemas de información y de programas de selección, inducción y capacitación de personal (Guerra, 2015)

En el sector público, los intereses generales son que los funcionarios públicos deben satisfacer el beneficio público siendo equitativo en el manejo correcto de los recursos públicos, la alta dirección y los funcionarios de todos los niveles tienen que estar involucrados en este proceso para resolver los riesgos y poder proporcionar seguridad razonable en el logro de la misión institucional (Argandoña, 2012).

El control interno en las entidades del sector público debe ser entendido dentro del contexto de las características específicas de estas organizaciones, es decir su enfoque para lograr objetivos sociales o políticos; la utilización de los fondos públicos; la importancia del ciclo presupuestario; la complejidad de su funcionamiento lo cual lleva a hacer un balance entre los valores tradicionales como la legalidad, integridad y transparencia, y los modernos valores gerenciales como eficiencia y eficacia) y el gran espectro correspondiente de su responsabilidad pública.

Las entidades públicas requieren del cumplimiento de muchas leyes y regulaciones, en éstas se ordenan la obtención de ingresos y ejecución de gastos con los dineros públicos y la forma de operar, entre ellos se puede citar la ley de presupuesto, tratados internacionales, ley general de contratación pública, leyes sobre la administración y gestión pública, ley de contabilidad, ley de derechos civiles y protección del medio ambiente, regulaciones sobre el manejo de los impuestos y acciones que eviten el fraude y la corrupción, que tanto mal le ha causado al patrimonio público, entre otros elementos que son fundamentales para el buen uso de los recursos.

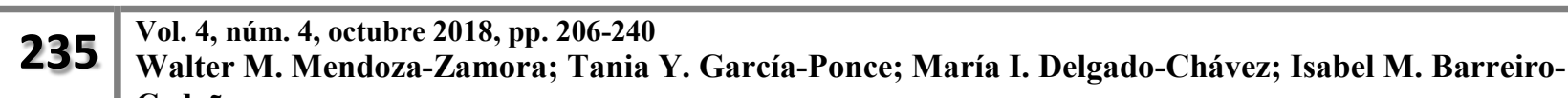
Cedeño
} 
Un sistema de control interno eficiente, no importa cuán bien se haya diseñado y esté operando el sistema de gestión de riesgos del ente público, no puede suministrar a la administración pública una seguridad absoluta respecto al logro de sus objetivos misionales, en lugar de ello, este componente reconoce que solo se puede obtener un nivel de seguridad razonable, pero el control interno no puede cambiar una administración inherentemente mala por una buen, además, los factores externos fuera de control de una entidad, como el factor político, pueden impactar la capacidad de alcanzar estos objetivos, consecuentemente un eficiente sistema de control interno reduce la probabilidad de no alcanzar los objetivos institucionales (Gámez, 2010).

El control interno incluirá las políticas y prácticas necesarias para asegurar una apropiada planificación y administración del talento humano de la institución, de manera que se garantice el desarrollo profesional y asegure la transparencia, eficacia y vocación de servicio; considerando que el talento humano es lo más valioso que posee cualquier institución, por lo que debe ser tratado y conducido de forma tal que se consiga su más elevado rendimiento (Koontz \& Heinz, 2009).

De cualquier forma, siempre habrá riesgo de que el control interno sea diseñado de manera deficiente o no opere tan eficientemente como se espera que funcione, dado que depende del juicio humano, está sujeto a fallas humanas tales como errores simples o equivocaciones; mala comprensión, descuido, fatiga, distracción, colusión, abuso o excesos, estas limitaciones impiden a la alta dirección tener una seguridad absoluta de que los objetivos sean alcanzados; por ello, la alta dirección necesita evaluar y actualizar continuamente los controles, informar de los cambios al personal, y dar ejemplo con la adhesión a estos controles.

Estimando una vez que el sistema está operando, se requiere de una previsión sobre una base de pruebas para ver si los controles previstos están operando como se planeó. Por esto el control

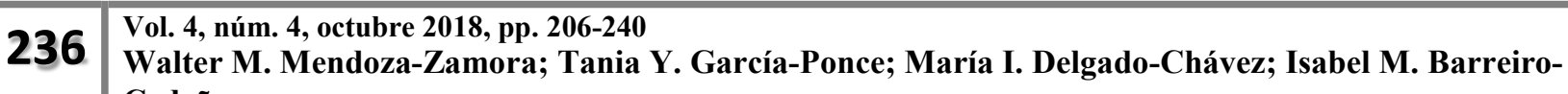
Cedeño
} 
interno no puede funcionar paralelamente al sistema, por estar estos íntimamente relacionados, es decir, funcionan como un todo, para lograr el objetivo establecido por la organización.

\section{Conclusiones.}

El Control Interno en las entidades del sector público debe ser entendido dentro del contexto de sus características específicas en comparación con las organizaciones privadas; es decir, tomando en cuenta a) su enfoque para lograr objetivos sociales o políticos; b) la utilización de los fondos públicos; c) la importancia del ciclo presupuestario y de planeamiento, y d) la complejidad de su funcionamiento. Esto significa hacer un balance entre los valores tradicionales como la legalidad, la integridad y la transparencia, presentes por su naturaleza en los asuntos públicos y los valores gerenciales modernos como la eficiencia y la eficacia.

Al mismo tiempo, esta evaluación del control interno nos permite obtener un conocimiento de las empresas e informar a la administración o a los responsables del gobierno corporativo, respecto a fallas o debilidades en el sistema de control interno, las cuales han sido identificadas en el transcurso de nuestra auditoría; esto brindará un valor agregado al cliente al contratar nuestros servicios como auditores.

Mejorando el desempeño de la ejecución de los recursos públicos, los mecanismos de control interno que implementen los Gestión administrativa reforzarán los sistemas administrativos vinculados al ciclo de gasto público, lo que redundará en mejores plazos para la formulación, aprobaciones, ejecución y rendición de cuentas de los recursos públicos asignados o generados, cuando se hable de procesos críticos de la entidad solo vincularán a las unidades que intervienen en

\footnotetext{
237 Vol. 4, núm. 4, octubre 2018, pp. 206-240
Walter M. Mendoza-Zamora; Tania Y. García-Ponce; María I. Delgado-Chávez; Isabel M. BarreiroCedeño
} 
todo aquello que tenga que ver directamente con uso de recursos, como son los procesos de compras, logística, almacenaje, entre otros.

Cabe indicar que el Control Interno debe ser diseñado desde la propia gestión, no por encima de las actividades, sino como parte del ciclo de gestión ya que al diseñarlo desde adentro, éste se vuelve parte integrada de los procesos de planificación, ejecución y seguimiento de la entidad. Además su concepción desde adentro tiene importantes implicaciones desde la perspectiva del costo ya que añadir procedimientos de control que están separados de los procedimientos existentes, aumenta los costos y se convierte en una carga que no genera valor, enfocándose en las operaciones existentes y en su contribución al Control Interno efectivo e integrando los diferentes controles en las actividades operativas básicas, la entidad puede evitar procedimientos y costos innecesarios.

Las entidades públicas requieren del cumplimiento de normativas (que dependiendo de las funciones asignadas o de los sistemas administrativos pueden ser abundantes y especializadas). En éstas, se reglamenta la gestión de la entidad y la forma de operar, los ejemplos incluyen la ley de presupuesto, los tratados internacionales, la ley general de contratación pública, las leyes sobre la administración y gestión pública, la ley de contabilidad, la ley de derechos civiles y protección del medio ambiente, las regulaciones sobre el manejo de los impuestos y las acciones que eviten el fraude y la corrupción.

Para acotar un poco más sobre El Control Interno al ser una herramienta de gestión debe ser implementada por las propias entidades del Estado, por ello, corresponde al titular y a los funcionarios la implementación y el funcionamiento del control interno en sus procesos, actividades, recursos y operaciones, orientando su ejecución al cumplimiento de sus objetivos.

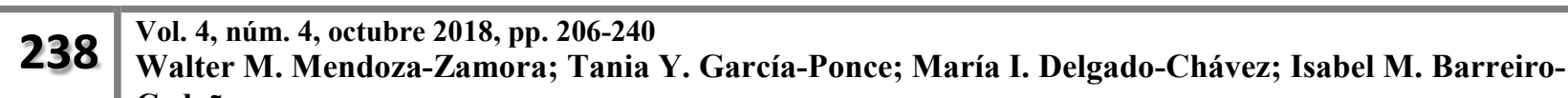
Cedeño
} 


\section{Bibliografía.}

Alvarado, J. (2012). El Control Interno de una Empresa Comercial. Guatemala: Prentice Hall.

Argandoña, M. (2012). Control Interno y Administración de Riesgo en la Gestión Pública. . Lima: Marketing Consultores S.A.

Brever, C. A. (2011). "Política, Estado y Administración PUblica". . Caracas, Venezuela: Editorial Ateneo.

Carrasco. (2011). investigación denominada Estudio Sobre Implementación de Gestión Basada en Procesos en Banco Estado. Santiago de Chile : s/.

Catágora, F. (2013). Sistemas y procedimientos contables. Venezuela: Editorial McGraw Hill.

Chacón, W. (2002). El control interno como herramienta fundamental contable y controladora de las organizaciones. Fulldocs.

Charry, J. A. (2013). El Control Interno en Diez Módulos en Entidades y Organismos del Estado, . Medellín, Colombia.: Editorial Diké.

Charry-Rodríguez, J. (2013). El control interno y los principios de evaluación de gestión en las entidades del Estado. Bogotá, Colombia.: Editorial Asociación colombiana de administradores.

Chiavenato, I. (2004.). Introducción a la Teoría General de la Administración. España: Séptima Edición, McGraw-Hill/ Interamericana, editores .

Estrada, M. (2010). Sistemas de control interno. Lima, Perú: Editorial Publicidad \& Matiz.

Fonseca, L. O. (2011). Sistemas de control interno. Lima, Perú: Editorial Publicidad \& Matiz.

Gámez, I. (2010). Control Interno en las áreas de aprovisionamiento de las empresas públicas de Andalucía. (Tesis doctoral). . España: Universidad de Málaga.

Gobernanza Democrática. (2011). El Control Interno en el sector Público. España: wordpress.

Gómez, M. (2010). Control Interno de las empresas públicas . España: Universidad de Málaga.

Guerra, M. (2015). Que es Gestión Administrativa, Origen, Evolución, Precursores y Principios. Quito: s/n.

Guerrero, A. (2008). "La Teoría de la Administración Pública". México: Editorial Harla México.

Herrera, R. (2015). Falla Control Interno en el Sector Público. México: s/n.

Koontz, H., \& Heinz, W. (2009). Administración, Una perspectiva global. México: McGraw-Hill.

Langrod, G. (2010). "Tratado de Ciencia Administrativa". Ed. Instituto de Estudios Administrativos -Escuela Nacional de Administración Pública . Madrid-España.: s/n. 
Leiva, D. (06 de Mayo de 2015). Marco conceptual del control interno. Obtenido de Minam: http://www.minam.gob.pe/comite/wpcontent/

Leonard, W. (2001). Evaluación de métodos y Eficiencia administrativa. . México: Editorial Diana.

Mantilla, S. A. (2008). Control interno de los nuevos instrumentos financieros. Bogotá, Colombia: Editorial Ecoe.

Martillo, L. (2008). Control interno de instrumentos financieros. Bogotá, Colombia: Editorial Ecoe.

Meigs, W., \& Larsen, J. (2004). Principios de Auditoría. . México: Segunda Edición. México. Editorial Diana. .

Mejía, G. B. (2006). Gerencia de procesos para el control interno. Bogotá, Colombia: Quinta edición, Editorial Ecoe.

Poch, R. (2000). Manual de Control Interno. Barcelona España. : Editorial Gestión 2000. Segunda Edición.

Pungitore, J. L. (2010). Sistemas administrativos y control interno. Barcelona, España.: Editorial Osmar D. Buyatti.

Ramón, J. G. (2001). El control interno en las Empresas privadas. quipukamayoc.

Reyes, H. J. (2012). "Política y Administración". México.: Serie Praxis INAP.

Rodriguez-Peñaherrera, C. (2000). "El Proceso de Reforma Administrativa en el Ecuador". SeminarioLatinoamericano de Aspectos Administrativos de la Planificación. Santiago de Chile: ONU-CEPAL. 